\title{
Pulse Baseline Wander Removal Using Wavelet Approximation
}

\author{
KQ Wang, LS Xu, L Wang, ZG Li, YZ Li \\ Department of Computer Science and Engineering \\ Harbin Institute of Technology, Harbin, China
}

\begin{abstract}
Pulse waveform is the key to the Traditional Chinese Pulse Diagnosis. However, its baseline wander introduced in the acquisition may result in misdiagnosis. What s more, recent advancements in the pulse variability analysis require more accuracy of baseline estimation. In this paper, a wavelet based cascaded adaptive filter (CAF) was presented to remove this drift. The CAF works in two stages. The first stage is a discrete Meyer wavelet approximation and the second stage is a cubic Spline estimation. The experimental results on 50 simulated and 200 real pulse signals demonstrate the powerful effect of CAF both in removing the baseline wander and in preserving the diagnostic information of pulse waveform, comparing with some traditional methods, such as cubic Spline estimation, morphology filter and Linear-phase FIR least-squares-error digital filter. In addition, this CAF is easy to be accomplished and needs no prior knowledge on pulse and its baseline drift.
\end{abstract}

\section{Introduction}

By means of modern signal processing technologies, Traditional Chinese Pulse Diagnosis (TCPD), one of the four diagnostic methods of TCM, is developing in the direction of keeping the distinctive features and combining with modern sciences and technologies. According to TCPD, pulse waveforms are used extensively for diagnosis, pathology, physiology and psychology researches [1-3].

We designed a pulse acquiring system, whose bandwidth is usually from $0.05 \mathrm{~Hz}$ to $100 \mathrm{~Hz}$ with almost linear response, causing no distortion to the pulse waveform. However, distortions may arise from subject $\mathrm{s}$ movement, respiration and so on during the pulse acquisition. The removal of these distortions is the first step in the pulse waveform processing. Especially, the baseline removal reemerges for further and more accurate analysis of pulse s and its parameters variability [4].

This paper is organized as follows. The related works on baseline removal is reviewed and their filtering performances are compared in Section 2. Section 3 depicts our brand new cascades adaptive filter. The effectiveness is illustrated both through simulated pulses in Section 4 and real typical pulses from our pulse database in Section 5. Finally, we draw some conclusions.

\section{Related works}

As a kind of one-dimensional physiological signal, pulse $\mathrm{s}$ baseline is something like other physiological signals . So far, there are some approaches on handling baseline drift of Electrocardiogram (ECG) and impedance cardiogram [5-6].

Nowadays, wavelets constitute an important and fairly new tool for signal processing. In this study, the wavelet approximation is applied as a tool to obtain a good approximation of baseline wander.

The wavelet approximation can eliminate the major part of baseline wander, but cannot put the baseline exactly to zero level. Therefore, we use the cubic Spline estimation to make the filtered pulse $\mathrm{s}$ baseline return exactly to zero level.

Forty-eight beats per minute are considered as the lowest heart rate to be processed. The pulse is considered to be periodic, and then the lowest frequency component of the pulse spectrum is considered as $0.8 \mathrm{~Hz}$. For a person, his pulse rate is four to five times of his respiration rate. The motion artifacts are also characterized by low-frequency components when the objects are quiet. Consequently, we set the cut-off frequency of removing baseline drift at $0.68 \mathrm{~Hz}$.

We designed the traditional linear-phase leastsquares-error FIR filter. But it did very little in canceling the baseline drift and might cause Gibbs phenomenon. The cubic Spline method assumes that the baseline fluctuation is little. The more the fluctuation is, the worse Spline estimation performs. For lacking of the reference signal, the adaptive of LMS filter and Wiener filter cannot achieve high performance. Thus, we only compare the FIR filter, morphology filter and wavelet filter s performances in removing the pulse s baseline. As shown in Figure 1, Sig 2 is the contaminated signal of Sig1. Sig 3 is the result of the morphological filter with the baseline 
drift being attenuated little. With a disk shaped structuring element sequence of 50 -sample points width, this morphological filter firstly performs opening operation and then performs closing operation for corrupted pulse. This filter can introduce some distortion to the pulse signal. Sig4 and Sig 5 are results processed by the 600 -order FIR filter with the cut-off frequency of $0.6 \mathrm{~Hz}$ and by the discrete Meyer Wavelet respectively. Figure 1(b) is the local enlargement of Figure 1(a). Compared with Sig1, the signal to noise ratio (SNR) of Sig4 and Sig5 are enhanced greatly. But Sig3 was distorted greatly. For the feature extraction and analysis, we hope all the onsets in every period of pulse are located in the same fiducial line. The onset is defined as the base point in every period of pulse. However, Sig 5 still remains a little baseline drift. We adapt the Spline estimation filter to rectify the distortion further. Under the high SNR condition, the Spline estimation s performance is satisfactory.

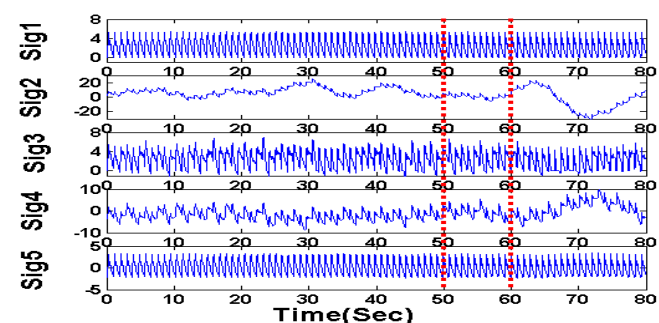

(a)

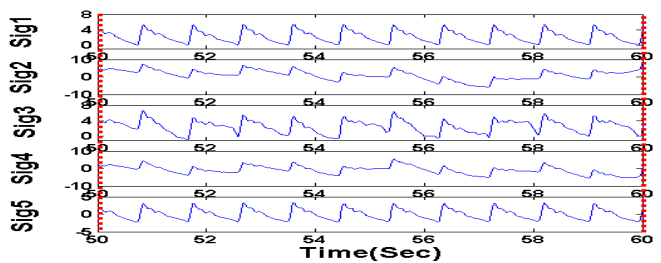

(b)

Fig. 1. Corrupted pulse and its filtered results by Morphology filter, FIR and Meyer wavelet.

Nevertheless, when the baseline is little, the wavelet filter may introduce some distortion. Figure2 showed that when the baseline is not high, Spline estimation is superior to wavelet filter. In Figure 2(a), baseline1 and baseline 3 are baselines filtered by wavelet filter and Spline filters respectively, while baseline 2 is the known baseline, which is constructed by filtering a random noise with a low pass filter at the $0.6 \mathrm{~Hz}$ cut-off frequency. The upper panel in Figure 2(b) is the case that the baseline s amplitude is little, while the lower panel is in the situation when the baseline is high. Both are come from the same clean pulse signal. Therefore, when the baseline wander is high, the wavelet filter performs better than Spline. But this is not the case when the signal and the baseline are both little. In Figure 2(c), the baseline is same, but the clean pulse signals are not. The clean pulse s amplitude in the lower row is ten times of the one in the lower row. So the energy ratio $(\boldsymbol{E R})$ of the clean signal to the baseline is the key to the baseline removal. Valdimir Shusterman noticed the non-ideal nature of the filter and he use the mean square error $(\boldsymbol{M S E})$ as a criterion to judge whether the baseline is small enough. He designed a two-step procedure for selective filter of ECG and removal of residual error with minimal distortion of cardiac complexes and tested the approach. $\boldsymbol{E R}$ is superior to $\boldsymbol{M S E}$, and can be applied to arbitrary length of signal [6]. Therefore, we applied the discrete Meyer wavelet to decompose the corrupted pulse signal to six levels. We compute the energy ratio of the first level approximation content of corrupted pulse signal to its sixth approximation.

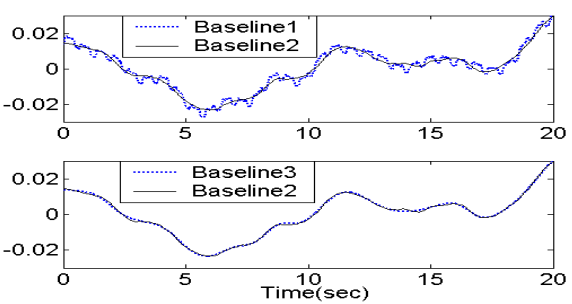

(a)

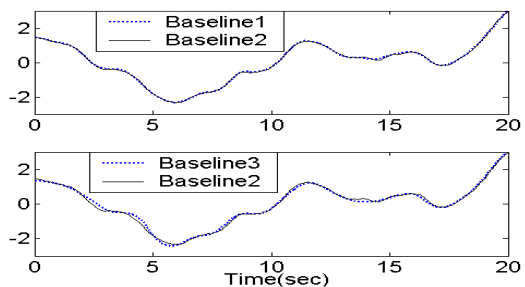

(b)
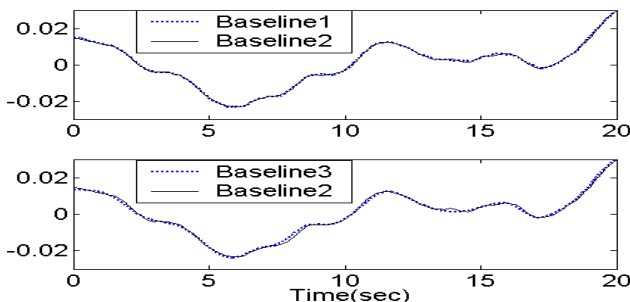

(c)

Fig. 2. The comparisons of Spline and wavelet filter during different baseline drift.

$$
E R=20 \log _{10} \frac{\operatorname{norm} 2(A 1-\operatorname{mean}(A 1))}{\operatorname{norm} 2(A 6-\operatorname{mean}(A 6))},
$$


Where, $\boldsymbol{A 1}$ is the first level approximation content of corrupted pulse signal, and $\boldsymbol{A 6}$ is the sixth level approximation content of corrupted pulse signal. Norm 2 means the two-order norm and mean $(A 1)$ means the average of $\boldsymbol{A} \boldsymbol{1}$.

After computing the $\boldsymbol{E R}$ of the signal, we judge if the corrupted signal needs to be filtered by the wavelet filter. From extensive experiments, we find that $\boldsymbol{E R}=\mathbf{5 0 d} \boldsymbol{b}$ is the best dividing between high and low baseline level. For the consideration of the accuracy, when the $\boldsymbol{E} \boldsymbol{R}$ of the corrupted pulse is more than $50 \mathrm{db}$, it can only be filtered by the Spline. Having analyzed the characters of pulse and its baseline wander and having compared many methods, we proposed a CAF filter.

\section{Cascaded wavelet filter}

The proposed CAF is depicted in Figure 3. The first stage is a discrete Meyer wavelet filter, and the second stage is a cubic Spline estimation filter. The primary input signal is pulse contaminated by baseline wander and this filter does not need any reference input as LMS adaptive filter does. Pulse1 is the output of the discrete Meyer wavelet filter. But Pulse1 still remains a little baseline contamination and needs to be corrected to the

Table 1 MSE of the CAF in filtering the simulated real baselines and typical pulses

\begin{tabular}{c|cllllllll}
\hline \multicolumn{1}{c}{ SNR $(\mathrm{db})$} & 10 & 5 & 0 & -5 & -10 & -15 & -20 \\
\hline \multirow{8}{*}{} & Taunt pulse & 0 & 0 & 0.01 & 0.03 & 0.1 & 0.3 & 0.3 \\
& Smooth pulse & 0.08 & 0.08 & 0.1 & 0.13 & 0.2 & 0.5 & 0.7 \\
& M S E & Normal pulse & 0.1 & 0.1 & 0.1 & 0.2 & 0.4 & 0.6 & 0.7 \\
& Slow pulse & 0.5 & 0.5 & 0.6 & 0.8 & 1.0 & 1.4 & 1.4 \\
& Rapid pulse & 0.01 & 0.02 & 0.02 & 0.04 & 0.1 & 0.3 & 0.3 \\
\hline
\end{tabular}

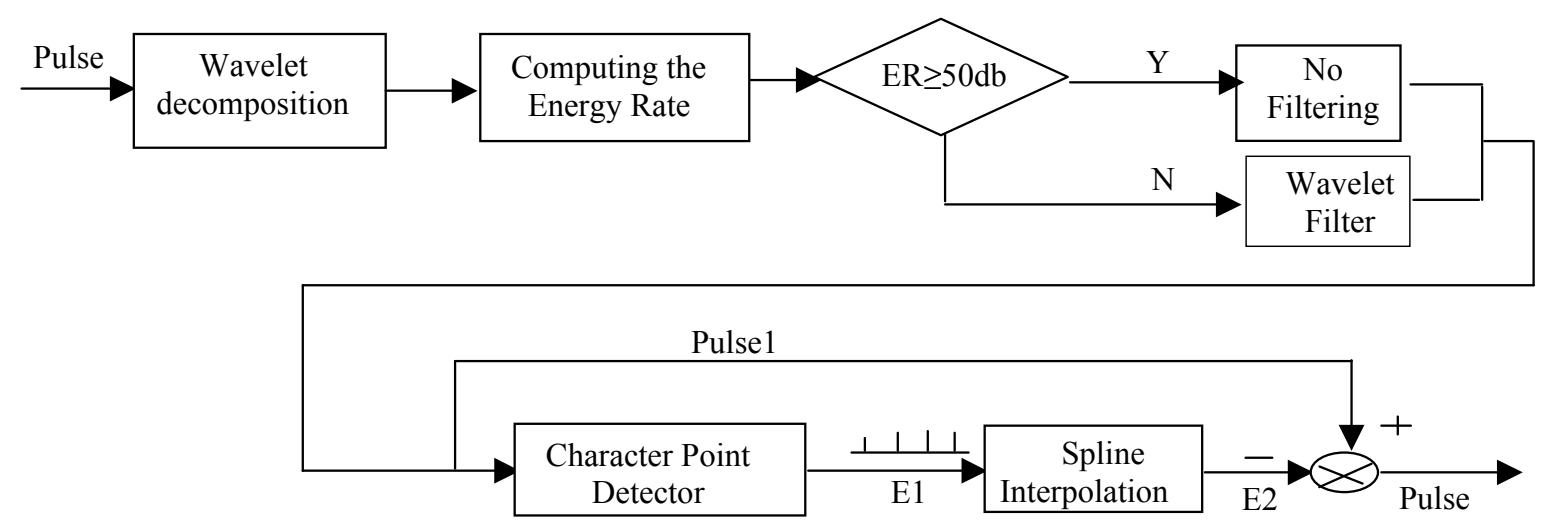

Fig.3 The cascaded adaptive filter 


\section{Experimental results for simulated signals}

In order to further analysis the performance of the CAF, we mixed 5 typical pulses and real baselines at different SNR. For emulating typical baseline wander introduced in the pulse recordings, our pulse sensor was attached to the wrist near the radial vessel so that the signal of subject $\mathrm{s}$ respiratory and body s motion artifacts can be acquired accurately. This signal from the transducer was amplified and conditioned in the same way as the pulse. The performances are listed in table 1. The 50 clean pulses are picked up from our pulse database, including Taunt Pulse, Smooth Pulse, Normal Pulse, Slow Pulse and Rapid Pulse. Every type of pulses includes 10 clean pulses. The Rapid Pulses pulse rate range from 120 to 110 pulse beats per minute and the Slow Pulses range from 53 to 49 , while the other pulses range from 62 to 85. The 10 baselines were all acquired from the 10 volunteers in the supine and sit position. The every 50 clean pulses plus the 10 baselines at several SNR, and then we choose the maximum among the MSEs and list them in Table1. We can find the MSE of the filter is not high even when the SNR is less than $-20 \mathrm{db}$, and when the pulse is Slow Pulse, the CAF s performance degraded.

\section{Experimental results for actual pulse records}
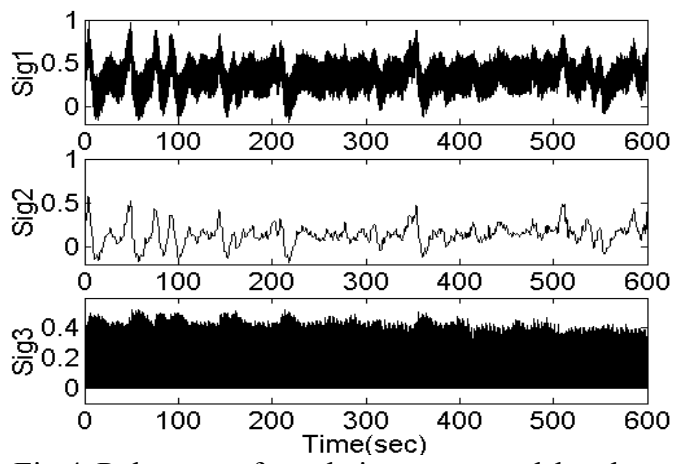

Fig.4 Pulse waveform being processed by the cascaded filter

In order to test the effect of this CAF filter, we use 200 typical pulse data from our pulse database. And we find $5 \%$ pulses have the ER more than 50db. In Figure 4, the Sig1 is 600 seconds pulse chosen from 8-hour sleeping monitoring database; Sig3 is the filtered signal; Sig 2 is the baseline extracted from the corrupted pulse. Having extracted the object $\mathrm{s}$ motion and respiration during sleeping, not only the clean pulse can be analyzed but also the baseline itself can be used to analyze sleeping, nerve and so on. What s more, this CAF filter does not need any reference input. The results are proved to be inspiring. This CAF filter is useful for the variability research of long-term physiology signal.

\section{Conclusions}

Estimation and correction of baseline wander is the basic problem for the physiological signal analysis. Recently, it is reemerged for more accurate analysis of these signals. Compared with Morphology filter, the cubic Spline method, the high-pass FIR filter, the wavelet procedure is adaptive, user-independent and performs better with respect to the others both in preserving the diagnostic information and in baseline wander removal.

We use the CAF filter in the 200 real corrupted pulses and find it is efficient and robust. The promising results we have obtained also encourages the use of this CAF technique to remove the baseline wander of other physiological signal.

\section{Acknowledgements}

This research was supported by the National Natural Science Foundation of China (90209020) the Multidiscipline Scientific Research Foundation of Harbin Institute of Technology (HIT.MD2001.36).

\section{References}

[1] Lu WA, Wang YY, Wang WK. Pulse analysis of patients with severe liver problems. IEEE Engineering in Medicine and Biology 1999; 18: 7375.

[2] Young ZY, Myeong HL, Kwang SS, Michael. Pulse type classification by varying contact pressure. IEEE Engineering in Medicine and Biology 2000; 19:106-110.

[3] Li SZ. Pulse Diagnosis 1985. Paradigm Press

[4] Wang KQ, Xu LS, Zhang D. TCPD based pulse monitoring and analyzing. ICMLC2002; Beijing

[5] Laguna R, Thakor P, Caminal NV. Adaptive baseline wander removal in the ECG: Comparative analysis with cubic spline technique. Computers in Cardiology 1992; 19: 143 -146.

[6] Vladimir S et al. Enhancing the precision of ECG baseline correction: selective filtering and removal of residual error. Computer and biomedical research 2000; 33: 144-160.

Corresponding author:

Lisheng $\mathrm{Xu}$

P.O.Box 332, Harbin Institute of Technology,

No.92, Xi Da Zhi Street, Harbin, Heilongjiang Province, China 150001

E-mail: lishengxu@hotmail.com 